B.P. Duggal*

\title{
On $(m, P)$-expansive operators: products, perturbation by nilpotents, Drazin invertibility
}

https://doi.org/10.1515/conop-2020-0120

Received February 14, 2021; accepted October 8, 2021

Abstract: A generalisation of $m$-expansive Hilbert space operators $T \in B(\mathcal{H})[18,20]$ to Banach space operators $T \in B(X)$ is obtained by defining that a pair of operators $A, B \in B(X)$ is $(m, P)$-expansive for some operator $P \in B(X)$ if $\triangle_{A, B}^{m}(P)=\left(I-L_{A} R_{B}\right)^{m}(P)=\sum_{j=0}^{m}(-1)^{j}\left(\begin{array}{c}m \\ j\end{array}\right) A^{j} P B^{j} \leq 0 ; L_{A}(X)=A X$ and $R_{B}(X)=X B$. Unlike $m$-isometric and $m$-left invertible operators, commuting products and perturbations by commuting nilpotents of $(m, I)$-expansive operators do not result in expansive operators: using elementary algebraic properties of the left and right multiplication operators, a sufficient condition is proved. For Drazin invertible $A$ and $B \in B(\mathcal{H})$, with Drazin inverses $A_{d}$ and $B_{d}$, a sufficient condition proving $\left(A_{d}, B_{d}\right) \wedge(A, B)$ is $(m-1, P)$ isometric (resp., $(m-1, P)$-contractive) for $m$ even (resp., $m$ odd) is given, and a Banach space analogue of this result is proved.

Keywords: Hilbert/Banach space, Left/right multiplication operator, $(m, P)$-expansive operators, $m$-left invertible/ $m$-isometric operators, product of operators, perturbation by nilpotents, Drazin invertible operators

MSC: 47A05, 47A55; Secondary47A11, 47B47

\section{Introduction}

Let $\mathcal{H}$ (resp., $X$ ) denote an infinite dimensional complex Hilbert (resp., Banach) space, and let $B(\mathcal{H})$ (resp., $B(X)$ ) denote the algebra of operators, i.e. bounded linear transformations, on $\mathcal{H}$ (resp., $X$ ) into itself. An operator $T \in B(\mathcal{H})$ is $m$-expansive for some positive integer $m$ if

$$
\triangle_{T^{\star}, T}^{m}(I)=\sum_{j=0}^{m}(-1)^{j}\left(\begin{array}{c}
m \\
j
\end{array}\right) T^{\star j} T^{j} \leq 0
$$

[20]; $T$ is m-hyperexpansive (resp., completely hyperexpansive) if $\triangle_{T^{*}, T}^{t}(I) \leq 0$ for all integers $1 \leq t \leq m$ (resp., for all positive integers $t$ ). Agler [1, Theorem 3.1] characterised subnormality with positivity of $\triangle_{T^{*}, T}^{m}(I)$ : $\triangle_{T^{*}, T}^{m}(I) \geq 0$ if and only if $\|T\| \leq 1$ and $T$ is subnormal. Operators $T$ such that $\triangle_{T^{*}, T}^{m}(I) \geq 0$ have been called $m$ contractive and operators $T$ such that $\triangle_{T^{*}, T}^{m}(I)=0$ are said to be $m$-isometric [2]. A study of $m$-isometries was initiated by Agler and Stankus in their series of papers [2-4] and further studies of the structure of this class of operators has since been carried out by a number of authors, amongst them [5-17]. Amongst $m$-expansive operators, 2-expansive operators stand out: 2-expansive operators are 2-hyperexpansive. A. Athavale studied completely hyperexpansive operators [18], and a study of $m$-expansive operators, including various of their avatars, has since been widely carried out in [19-23].

^Corresponding Author: B.P. Duggal: University of Niš, Faculty of Sciences and Mathematics, P.O. Box 224, 18000 Niš, Serbia, E-mail: bpduggal@yahoo.co.uk 
For $A, B \in B(X)$, let $L_{A}$ and $R_{B} \in B(B(X))$ denote respectively the operators

$$
L_{A}(X)=A X \text { and } R_{B}(X)=X B
$$

of left multiplication by $A$ and right multiplication by $B$. If we define the elementary operator $\triangle_{A, B} \in B(B(X))$ by

$$
\triangle_{A, B}(X)=\left(I-L_{A} R_{B}\right)(X)=X-A X B
$$

then

$$
\begin{aligned}
& \triangle_{A, B}^{m}(X)=\left(I-L_{A} R_{B}\right)^{m}(X)=\sum_{j=0}^{m}(-1)^{j}\left(\begin{array}{c}
m \\
j
\end{array}\right)\left(L_{A} R_{B}\right)^{j}(X) \\
= & \sum_{j=0}^{m}(-1)^{j}\left(\begin{array}{c}
m \\
j
\end{array}\right) A^{j} X B^{j} .
\end{aligned}
$$

Recall [24] that a pair of operators $A, B \in B(X)$ is $(m, X)$-left invertible for some operator $X \in B(\mathcal{H})$ if $\triangle_{A, B}^{m}(X)=0$. Generalising to $m$-expansive operators, we say that a pair $(A, B) \in B(X)$ is $(m, X)$-expansive for an $X \in B(X)$ if $\triangle_{A, B}^{m}(X) \leq 0$. Trivially, every $(m, X)$-left invertible pair $(A, B)$ is $(m, X)$-expansive; $(m, X)$ expansive pairs, however, fail in general to satisfy properties satisfied by $(m, X)$-left invertible pairs (even if one limits oneself to Hilbert space pairs $\left(T^{\star}, T\right)$ ). Thus $(A, B) \in(m, X)$-left invertible implies $(A, B) \in(n, X)$ left invertible for all integers $n \geq m$ : $\left(T^{\star}, T\right) \in(m, X)$-expansive, $T \in B(\mathcal{H})$, fails to imply $\left(T^{\star}, T\right) \in(m+1, X)$ expansive. Since

$$
\triangle_{A^{n}, B^{n}}^{m}(X)=\left(I-L_{A}^{n} R_{B}^{n}\right)^{m}(X)=\left\{I+L_{A} R_{B}+\cdots+L_{A}^{n-1} R_{B}^{n-1}\right\}^{m} \triangle_{A, B}^{m}(X),
$$

$(A, B) \in(m, X)$-left invertible implies $\left(A^{n}, B^{n}\right) \in(m, X)$-left invertible for all integers $n \geq m$, and if $A=$ $B^{\star}=T \in B(\mathcal{H})$ then $(A, B) \in(m, X)$-expansive for an $X \in B(\mathcal{H})$ implies $\left(A^{n}, B^{n}\right) \in(m, X)$-expansive for all integers $n \geq m[20,24]$. The passage of the $(m, X)$-expansive property from $(A, B)$ to $\left(A^{n}, B^{n}\right)$ however fails for general $(A, B)$, and a sufficient condition here is the "existence of certain partial hyperexpansive sequences" (see Theorem 2.6, infra).

Richter [25, Lemma 1] observed that $(2, I)$-expansive Hilbert space operators are $(2, I)$-hyperexpansive (see also Athavale [18]; see $\mathrm{Gu}$ [26] for the Banach space analogue). This property is inherited by $(2, X)$ expansive Hilbert space pairs $\left(T^{\star}, T\right)$ for $X \geq 0$. We start in this paper by proving that: if $(A, B) \in\left\{\left(m_{0}, P\right)\right.$ cotractive $\} \wedge\left\{\left(m_{0}+2\right)\right.$-expansive $\} \wedge\{(m, P)$-expansive $\}$, where $A, B, P \in B(\mathcal{H}), m_{0} \geq 0$ and $m>m_{0}+2$ are integers, and the triplet $\left(A, B, \triangle_{A, B}^{S}(P)\right)$ satisfies an "order preserving property" (of type: if $\triangle_{A, B}^{s}(P) \geq 0$ for $m_{0}+2 \leq s \leq m$, then $\left(L_{A} R_{B}\right)^{t}\left(\triangle_{A, B}^{S_{0}}(P)\right) \geq 0$ for all positive integers $t$ - see Theorem 2.2 below), then $(A, B) \in(r, P)$-expansive for all integers $m_{0}+1 \leq r \leq m$. For invertible $(A, B)$ such that both the triplets $\left(A, B, \triangle_{A, B}^{s}(P)\right)$ and $\left(A^{-1}, B^{-1}, \triangle_{A, B}^{s}(P)\right)$ preserve order for $s=0, m-1, m$ and $(A, B) \in(m, P)$-expansive, we prove that $(A, B) \wedge\left(A^{-1}, B^{-1}\right) \in(n, P)$-isometric for all $n \geq m-1$ if $m$ is even and $(A, B) \wedge\left(A^{-1}, B^{-1}\right) \in(m-1, P)$ contractive if $m$ is odd. An analogous result holds for Drazin invertible pairs $(A, B)$.

If $A_{i}, B_{i}, X \in B(X)$ are such that $\left(A_{i}, B_{i}\right) \in\left(m_{i}, X\right)$-left invertible ( $\left.i=1,2\right), A_{1}$ commutes with $A_{2}$ and $B_{1}$ commutes with $B_{2}$, then the products pair $\left(A_{1} A_{2}, B_{1} B_{2}\right)$ is $\left(m_{1}+m_{2}-1, X\right)$-left invertible; again, if $N \in B(X)$ is an $n$-nilpotent which commutes with $A_{1}$ and $B_{1}$, then $\left(A_{1}, B_{1}\right) \in(m, X)$-left invertible implies $\left(A_{1}+N, B_{1}+\right.$ $N) \in\left(m_{1}+2 n-2\right)$-left invertible (see [5, 16, 24, 27]). These properties do not transfer to $(m, X)$-expansive operators. We prove that a sufficient condition for a successful transfer of these properties to $(m, X)$-expansive operators is the existence of "partial expansive sequences". The existence of such sequences for left invertible pairs is a (trivial) consequence of the structure of such pairs.

We prove our results, including any complementary results, in the following two sections. In addition to the notation and terminology already introduced, any further notation/terminology will be explained at the first instance of occurence of such notation/terminology. 


\section{$2(m, P)$-hyperexpansive operators on Hilbert and Banach spaces.}

An important, and well studied, class of $(m, X)$-expansive operators in $B(\mathcal{H})$ is that of $m$-isometric operators, where $S \in B(\mathcal{H})$ is $m$-isometric,equivalently $\left(S^{\star}, S\right) \in(m, I)$-isometric, if

$$
\triangle_{S^{*}, S}^{m}(I)=\left(I-L_{S^{*}} R_{S}\right)^{m}=\sum_{j=0}^{m}(-1)^{j}\left(\begin{array}{c}
m \\
j
\end{array}\right) S^{\star j} S^{j}=0
$$

(see $[2,5-8,13,16,28]$ for further references). It is clear that $m$-isometric operators are $(m, I)$-left invertible operators. Since $\triangle_{A, B}^{n}(X)=\triangle_{A, B}^{n-m}\left(\triangle_{A, B}^{m}(X)\right), \triangle_{A, B}^{0}(X)=X$, for all integers $n \geq m$, it follows that

$$
(A, B) \in(m, X) \text { - left invertible } \Rightarrow(A, B) \in(n, X) \text { - left invertible }
$$

for all integers $n \geq m$ [24, Proposition 2.1]. Let $[A, B]=A B-B A$ denote the commutant of the operators $A, B \in B(X)$. If $\left(A_{i}, B_{i}\right) \in B(X), i=1,2$, are such that $\left[A_{1}, A_{2}\right]=\left[B_{1}, B_{2}\right]=0$ and $\left(A_{i}, B_{i}\right) \in\left(m_{i}, X\right)$-left invertible, then

$$
\begin{aligned}
& \triangle_{A_{1} A_{2}, B_{1} B_{2}}^{m_{1}+m_{2}-1}(X)=\left[L_{A_{2}} \triangle_{A_{1}, B_{1}} R_{B_{2}}+\triangle_{A_{2}, B_{2}}\right]^{m_{1}+m_{2}-1}(X) \\
= & \sum_{j=0}^{m_{2}-1}\left(\begin{array}{c}
m_{1}+m_{2}-1 \\
j
\end{array}\right)\left(L_{A_{2}} R_{B_{2}}\right)^{m_{1}+m_{2}-1-j} \triangle_{A_{2}, B_{2}}^{j}\left(\triangle_{A_{1}, B_{1}}^{m_{1}+m_{2}-1-j}(X)\right)+ \\
+ & \sum_{j=m_{2}}^{m_{1}+m_{2}-1}\left(\begin{array}{c}
m_{1}+m_{2}-1 \\
j
\end{array}\right)\left(L_{A_{2}} R_{B_{2}}\right)^{m_{1}+m_{2}-1-j} \triangle_{A_{1}, B_{1}}^{m_{1}+m_{2}-1-j}\left(\triangle_{A_{2}, B_{2}}^{j}(X)\right) \\
= & 0
\end{aligned}
$$

i.e., $\left(A_{1} A_{2}, B_{1} B_{2}\right) \in\left(m_{1}+m_{2}-1, X\right)$-left invertible [24, Remark 2.3]. Again, if $A, B, N_{1} \in B(X), N_{1}$ is $n_{1}$ nilpotent and $\left[B, N_{1}\right]=0$, then

$$
\begin{aligned}
& \triangle_{A, B+N_{1}}^{m+n_{1}-1}(X)=\left[\triangle_{A, B}+L_{A} R_{N_{1}}\right]^{m+n_{1}-1}(X) \\
= & \sum_{j=0}^{n_{1}-1}\left(\begin{array}{c}
m+n_{1}-1 \\
j
\end{array}\right)\left(L_{A} R_{N_{1}}\right)^{j} \triangle_{A, B}^{m+n_{1}-1-j}(X)+ \\
+ & \sum_{j=n_{1}}^{m+n_{1}-1}\left(\begin{array}{c}
m+n_{1}-1 \\
j
\end{array}\right) \triangle_{A, B}^{m+n_{1}-1-j}\left(L_{A} R_{N_{1}}\right)^{j}(X) \\
= & 0,
\end{aligned}
$$

since $\triangle_{A, B}^{m+n_{1}-1-j}(X)=0$ for all $j \leq n_{1}-1$ (implies $m+n_{1}-1-j \geq m$ ) and $N_{1}^{j}=0$ for all $j \geq n_{1}$. Again, if $N_{2}$ is an $n_{2}$-nilpotent which commutes with $A$, then

$$
\begin{aligned}
\triangle_{A+N_{2}, B+N_{1}}^{m+n_{1}+n_{2}-2}(X) & =\left[\triangle_{A, B+N_{1}}+L_{N_{2}} R_{B+N_{1}}\right]^{m+n_{1}+n_{2}-2}(X) \\
& =\sum_{j=0}^{n_{2}-1}\left(\begin{array}{c}
m+n_{1}+n_{2}-2 \\
j
\end{array}\right)\left(L_{N_{2}} R_{B+N_{1}}\right)^{j} \triangle_{A, B+N_{1}}^{m+n_{1}+n_{2}-2-j}(X)+ \\
& +\sum_{j=n_{2}}^{m+n_{1}+n_{2}-2}\left(\begin{array}{c}
m+n_{1}+n_{2}-2 \\
j
\end{array}\right) \triangle_{A, B+N_{1}}^{m+n_{1}+n_{2}-2-j}\left(L_{N_{2}} R_{B+N_{1}}\right)^{j}(X) \\
& =0,
\end{aligned}
$$

since $\triangle_{A, B+N_{1}}^{m+n_{1}+n_{2}-2-j}(X)=0$ for all $j \leq n_{2}-1$ (implies $m+n_{1}+n_{2}-2-j \geq m+n_{1}-1$ ) and $N_{2}^{j}=0$ for all $j \geq n_{2}$ (cf. [24]).

These results fail for (non $(m, X)$-left invertible) $(m, X)$-expansive operators. 
Example 2.1. If we let $T=\alpha I, \alpha$ some complex number with modulus greater then one and $I$ the identity of $B(\mathcal{H})$, then

$$
\triangle_{T^{\star}, T}^{m}(I)=\sum_{j=0}^{m}(-1)^{j}\left(\begin{array}{c}
m \\
j
\end{array}\right)|\alpha|^{2 j}=\left(1-|\alpha|^{2}\right)^{m} I \leq 0
$$

for all odd positive integers $m$, hence $\left(T^{\star}, T\right) \in(2 n+1, I)$-expansive for all non-negative integers $n$. However, $\left(T^{\star}, T\right) \notin(2 n, I)$-expansive for any integer $n \geq 1$. Let $S=I$. Then, trivially, $S$ is isometic, hence $\left(S^{\star}, S\right) \in(2, I)$ isometric. Trivially, $[S, T]=0$ and $\left(S^{\star} T^{\star}, S T\right) \notin(2 n+2, I)$-expansive for any non-negative integer $n$.

Before going on to consider our next example (showing that the result on perturbation by commuting nilpotents does not extend from $(m, X)$-left invertible operators to $(m, X)$-expansive operators), we prove a generalisation of Richter's result [25, Lemma 1] that 2-expansive operators are 2-hyperexpansive. Given Hilbert space operators $A_{i}, P \in B(\mathcal{H}), i=1,2$, let $X_{S}=X_{S}\left(A_{1}, A_{2}, P\right)$ be the operator $X_{S}=\triangle_{A_{1}, A_{2}}^{s}(P)$ for some nonnegative integer $s$. (Thus $X_{0}=P$.) We say that the triplet $\left(A_{1}, A_{2}, X_{S}\right)$ preserves order if $L_{A_{1}}^{t} R_{A_{2}}^{t}\left(X_{S}\right) \geq 0$ (resp., $L_{A_{1}}^{t} R_{A_{2}}^{t}\left(X_{S}\right) \leq 0$ ) for all integers $t \geq 1$ whenever $X_{S} \geq 0$ (resp., $\left.X_{S} \leq 0\right)$. (Indeed, what we are saying here is that an application of powers of the elementary operator $E_{A, B}=L_{A} R_{B}$ preserves the positivity, or otherwise, of our operator $X_{S}$ : it is however convenient to shorten it to "triplet $\left(A, B, X_{S}\right)$ preserves order".) Order preserving triplets occur naturally. Thus, if $A, P \in B(\mathcal{H})$ with $P \geq 0$, then $A^{\star t} P A^{t} \geq 0$ for all positive integers $t$; more generally, if $A \in(m, P)$-expansive (resp., $A \in(m, P)$-contractive), then $A^{\star t} \triangle_{A^{\star}, A}^{m}(P) A^{t} \leq 0$ (resp., $\left.A^{\star t} \triangle_{A_{1}, A_{2}}^{m}(P) A^{t} \geq 0\right)$ for all positive integers $t$ (and the triplet $\left(A^{\star}, A, \triangle_{A^{\star}, A}^{m}(P)\right)$ is an order preserving triplet). Again, if $A_{1}, A_{2}$ and $P \in B(\mathcal{H})$ are such that $\left(A_{1}, A_{2}\right) \in(m, P)$-expansive for all $m \geq t$ for some positive integer $t$, then for all integers $s \geq t$,

$$
\begin{aligned}
& 0 \geq \triangle_{A_{1}, A_{2}}^{s+1}(P)=A_{1} \triangle_{A_{1}, A_{2}}^{s}(P) A_{2}-\triangle_{A_{1}, A_{2}}^{s}(P) \\
\Rightarrow & L_{A_{1}}^{t} R_{A_{2}}^{t} \triangle_{A_{1}, A_{2}}^{s}(P) \leq \triangle_{A_{1}, A_{2}}^{s}(P) \leq 0
\end{aligned}
$$

for all positive integers $t$ (so that the triplet $\left(A_{1}, A_{2}, \triangle_{A_{1}, A_{1}}^{t}(P)\right)$ preserves order).

Theorem 2.2. Let $A, B, P \in B(\mathcal{H})$ be such that $(A, B)$ is $\left(m_{0}, P\right)$-contractive for some non-negative integer $m_{0}$ (thus: $P \geq 0$ if $m_{0}=0$ ),

$$
(A, B) \in\left\{\left(m_{0}+2, P\right)-\text { expansive }\right\} \wedge\{(m, P)-\text { expansive }\}
$$

for some integer $m>m_{0}+2$ and the triplet $\left(A, B, \triangle_{A, B}^{r}(P)\right), m_{0}+2 \leq r \leq m$, preserve order. Then

$$
\triangle_{A, B}^{r}(P) \leq 0 \text { for all } m_{0}+1 \leq r \leq m .
$$

Proof. Define the operator $X \in B(\mathcal{H})$ by $X=\triangle_{A, B}^{m_{0}}(P)$; then $X \geq 0$. If we now let

$$
\tilde{\triangle}_{A, B}(S)=\left(L_{A} R_{B}-I\right)(S)=-\triangle_{A, B}(S)
$$

then

$$
\begin{aligned}
(A, B) \in\left(m_{0}+2, P\right)-\text { expansive } \Longleftrightarrow \triangle_{A, B}^{m_{0}+2}(P) & =\triangle_{A, B}^{2}(X) \leq 0 \\
& \Longleftrightarrow A^{2} X B^{2}-2 \tilde{\triangle}_{A, B}(X)-X \leq 0 \\
& \Longleftrightarrow A^{2} X B^{2} \leq 2 \tilde{\triangle}_{A, B}(X)+X,
\end{aligned}
$$

and this, since the triplet $\left(A, B, \triangle^{2} A, B(X)\right)$ preserves order, implies

$$
\begin{aligned}
& 0 \geq A^{3} X B^{3}-2 A \tilde{\triangle}_{A, B}(X) B-A X B \Rightarrow A^{3} X B^{3}-3 \tilde{\triangle}_{A, B}(X)-X \leq 0 \\
\Rightarrow & A^{3} X B^{3} \leq 3 \tilde{\triangle}_{A, B}(X)+X .
\end{aligned}
$$

Using an induction argument, it follows that

$$
A^{n} X B^{n} \leq n \tilde{\triangle}_{A, B}(X)+X \Longleftrightarrow \tilde{\triangle}_{A, B}(X) \geq \frac{1}{n}\left\{A^{n} X B^{n}-X\right\}
$$


for all posiitve integers $n$. Since $X \geq 0$ implies $\left(L_{A} R_{B}\right)^{n}(X) \geq 0$, letting $n$ go to infinity in the above we have

$$
\tilde{\triangle}_{A, B}(X) \geq 0 \Longleftrightarrow \triangle_{A, B}(X)=\triangle_{A, B}^{m_{0}+1}(P) \leq 0 .
$$

The hypothesis

$$
\begin{aligned}
& \tilde{\triangle}_{A, B}^{2}(X)=\triangle_{A, B}^{2}(X) \leq 0 \Longleftrightarrow A \tilde{\triangle}_{A, B}(X) B-\tilde{\triangle}_{A, B}(X) \leq 0 \\
\Rightarrow & A^{2} \tilde{\triangle}_{A, B}(X) B^{2}-A \tilde{\triangle}_{A, B}(X) B \leq 0 \\
\cdots & \\
\Rightarrow & A^{n} \tilde{\triangle}_{A, B}(X) B^{n}-A^{n-1} \tilde{\triangle}_{A, B}(X) B^{n-1} \leq 0
\end{aligned}
$$

for all integers $n \geq 1$. Hence

$$
A^{n} \tilde{\triangle}_{A, B}(X) B^{n} \leq A^{n-1} \tilde{\triangle}_{A, B}(X) B^{n-1} \leq \cdots \leq A \tilde{\triangle}_{A, B}(X) B \leq \tilde{\triangle}_{A, B}(X)(\geq 0),
$$

and $\left\{A^{n} \tilde{\triangle}_{A, B}(X) B^{n}\right\}_{n \geq 0}$ is a bounded below decreasing sequence of non-negative operators. Consequently, the sequence converges to a non-negative operator, say $Q$.

The hypothesis $(A, B) \in(m, P)$-expansive also implies that

$$
\triangle_{A, B}^{m}(P)=\triangle_{A, B}^{m-m_{0}}(X)=-A \triangle_{A, B}^{m-m_{0}-1}(X) B+\triangle_{A, B}^{m-m_{0}-1}(X) \leq 0 ;
$$

hence, since the triplet $\left(A, B, \triangle_{A, B}^{m-m_{0}}(X)\right)$ preserves order,

$$
\triangle_{A, B}^{m-m_{0}-1}(X) \leq A \triangle_{A, B}^{m-m_{0}-1}(X) B \leq \cdots \leq A^{n} \triangle_{A, B}^{m-m_{0}-1}(X) B^{n}
$$

for all integers $n \geq 0$. Since

$$
\begin{aligned}
A^{n} \triangle_{A, B}^{m-m_{0}-1}(X) B^{n} & =\sum_{j=0}^{m-m_{0}-2}(-1)^{j}\left(\begin{array}{c}
m-m_{0}-2 \\
j
\end{array}\right) A^{n+j} \triangle_{A, B}(X) B^{n+j} \\
& =-\sum_{j=0}^{m-m_{0}-2}(-1)^{j}\left(\begin{array}{c}
m-m_{0}-2 \\
j
\end{array}\right) A^{n+j} \tilde{\triangle}_{A, B}(X) B^{n+j},
\end{aligned}
$$

letting $n \longrightarrow \infty$ we have

$$
\lim _{n \longrightarrow \infty} A^{n} \triangle_{A, B}^{m-m_{0}-1}(X) B^{n}=-\sum_{j=0}^{m-m_{0}-2}(-1)^{j}\left(\begin{array}{c}
m-m_{0}-2 \\
j
\end{array}\right) Q=0 .
$$

Already we have

$$
\lim _{n \longrightarrow \infty} \triangle_{A, B}^{m-m_{0}-1}(X) \leq \lim _{n \longrightarrow \infty} A^{n} \triangle_{A, B}^{m-m_{0}-1}(X) B^{n} ;
$$

hence

$$
\triangle_{A, B}^{m-m_{0}-1}(X) \leq 0\left(\Longleftrightarrow \triangle_{A, B}^{m-1}(P) \leq 0\right) .
$$

Starting next with $\triangle_{A, B}^{m_{0}+2}(P) \wedge \triangle_{A, B}^{m-1}(P) \leq 0$, and repeating the argument, we obtain $\triangle_{A, B}^{m-2}(P) \leq 0$, and consequentlly that $\triangle_{A, B}^{r}(P) \leq 0$ for all $m_{0}+1 \leq r \leq m$.

An example of operators satisfying the order preservation property of Theorem 2.2 is provided by $\left(T^{\star}, T\right)$, $T \in B(\mathcal{H})$, such that $\left(T^{\star}, T\right) \in\left\{\left(m_{0}, P\right)\right.$-contractive $\} \wedge\left\{\left(m_{0}+2, P\right)\right.$-expansive $\} \wedge\{(m, P)$-expansive $\}$ for some operator $P \in B(\mathcal{H})$ and integers $2 \leq m_{0}+2<m$. Then $\triangle_{T^{*}, T}^{r}(P) \leq 0$ for all $m_{0}+1 \leq r \leq m$. In particular:

Corollary 2.3. Given operators $T, P \in B(\mathcal{H}), P \geq 0$, if $\left(T^{\star}, T\right) \in\{(2, P)$-expansive $\} \wedge\{(m, P)$-expansive $\}$ for some integer $m \geq 2$, then $\left(T^{\star}, T\right) \in(m, P)$-hyperexpansive. 
In addition to generalising the result that 2-expansive $B(\mathcal{H})$ operators are 2-hyperexpansive [18, 25], Theorem 2.2 generalises [19, Theorem 2.1] (by dispensing with the hypothesis that $\left(T^{\star}, T\right) \in(1, P)$-expansive - [19] considers the case $m_{0}=0-$ and by proving that $\left(T^{\star}, T\right)$ is $m$-hyperexpansive). Observe that

$$
\left(T^{\star}, T\right) \in(m, I)-\text { expansive } \Longleftrightarrow \sum_{j=0}^{m}(-1)^{j}\left(\begin{array}{c}
m \\
j
\end{array}\right)\left\|T^{j} x\right\|^{2} \leq 0
$$

for all $x \in \mathcal{H}$. A generalisation of $(m . P$ )-expansive operators to Banach space operators is obtained (following Bayart's [7] definition of Banach space $m$-isometric operators) as follows: $T \in B(\mathcal{X})$ is $(m, P)$-expansive for some operator $P \in B(X)$ if

$$
T \in \triangle_{p}^{m}(P, T, x)=\sum_{j=0}^{m}(-1)^{j}\left(\begin{array}{c}
m \\
j
\end{array}\right)\left\|P T^{j} x\right\|^{p} \leq 0
$$

for some integer $p \geq 1$ and all $x \in X[19,21]$. A version of Theorem 2.2 holds for $(m, P)$-expansive Banach space operators.

The following theorem, generalising corresponding results from $[19,21]$, considers the case $m_{0}=0$ of Theorem 2.2. Let $T \in \triangle_{p}^{m}(P, T, x)$ denote $T \in B(X)$ is ( $\left.m, P\right)$-expansive in the sense of (definition) (4).

Theorem 2.4. If an operator $T \in B(X)$ is such that

$$
T \in\left\{\triangle_{p}^{2}(P, T, x)\right\} \wedge\left\{\triangle_{p}^{m}(P, T, x)\right\}, m>2,
$$

for some integer $p \geq 1$ and operator $P \in B(X)$, then $T \in \triangle_{p}^{r}(P, T, x)$ for all $1 \leq r \leq m$ (i.e., $T$ is $(m, P)$ hyperexpansive in the sense of (4)).

Proof. The proof below being similar to that of Theorem 2.2, we shall be economical. If we let $\tilde{\triangle}_{p}(P, T, x)=$ $-\triangle_{p}(P, T, x)$, then $\triangle_{p}^{2}(P, T, x) \leq 0$ implies

$$
\left\|P T^{2} x\right\|^{p} \leq 2 \tilde{\triangle}_{p}(P, T, x)+\|P x\|^{p},
$$

and hence that

$$
\left\|P T^{n} x\right\|^{p} \leq n \tilde{\triangle}_{p}(P, T, x)+\|P x\|^{p} .
$$

This implies

$$
\tilde{\triangle}_{p}(P, T, x)=\lim _{n \longrightarrow \infty} \tilde{\triangle}_{p}(P, T, x) \geq \lim _{n \longrightarrow \infty} \frac{1}{n}\left\{\left\|P T^{n} x\right\|^{p}-\|P x\|^{p}\right\} \geq 0
$$

Since

$$
\left\|P T^{n} x\right\|^{p} \leq\left\|P T^{n-1} x\right\|^{p} \leq \cdots \leq\|P T x\|^{p} \leq\left\|P T^{n} x\right\|^{p}
$$

for all $x \in X$,

$$
\lim _{n \longrightarrow \infty}\left\|P T^{n} x\right\|^{p}=c
$$

for some scalar $c=c(x) \geq 0$. Furthermore, $\triangle_{p}^{2}(P, T, x) \leq 0$ implies $\tilde{\triangle}_{p}(P, T, T x)-\tilde{\triangle}_{p}(P, T, x) \leq 0$ and this in turn implies

$$
(0 \leq) \tilde{\triangle}_{p}(P, T, x) \geq \tilde{\triangle}_{p}(P, T, T x) \geq \cdots \geq \tilde{\triangle}_{p}\left(P, T, T^{n} x\right),
$$

where

$$
\tilde{\triangle}_{p}\left(P, T, T^{n} x\right)=\left\|P T^{n+1} x\right\|^{p}-\left\|P T^{n} x\right\|^{p} .
$$

Trivially,

$$
\triangle_{p}^{m}(P, T, x)=-\triangle_{p}^{m-1}(P, T, T x)+\triangle_{p}^{m-1}(P, T, x)(\leq 0) ;
$$

hence,

$$
\triangle_{p}^{m-1}(P, T, x) \leq \triangle_{p}^{m-1}\left(P, T, T^{n} x\right)
$$


for integers $n \geq 1$, therefore

$$
\begin{aligned}
\lim _{n \longrightarrow \infty} \triangle_{p}^{m-1}(P, T, x) & \leq \lim _{n \longrightarrow \infty} \sum_{j=0}^{m}(-1)^{j}\left(\begin{array}{c}
m \\
j
\end{array}\right)\left\|P T^{n+j} x\right\|^{p} \\
& =\sum_{j=0}^{m}(-1)^{j}\left(\begin{array}{c}
m \\
j
\end{array}\right) c=0,
\end{aligned}
$$

i.e., $\triangle_{p}^{m-1}(P, T, x) \leq 0$. The proof now follows from a finite repetition of the argument.

Next, we give our example on perturbation by commuting nilpotents.

Example 2.5. (i) If $T \in B(\mathcal{H})$ is an m-expansive operator, i.e. $\left(T^{\star}, T\right) \in(m, I)$-expansive, and $N \in B(\mathcal{H})$ is an $n$-nilpotent which commutes with $T$, then the example of the $m$-isometric operators dictates that, if at all then, $T+N$ is an $(m+2 n-2)$-expansive operator. Since $[T,-N]=0$, it follows that if $T+N$ is $(m+2 n-2)$-expansive, then $T(=T+N-N)$ is $(m+4 n-4)$-expansive also. Indeed, repeating the argument, alternating between perturbation by $N$ and $-N, T$ is $(m+2 n e-2 e)$-expansive for every non-negative even integer $e$. Assuming now that $m=2$, it follows that if $T$ is 2-expansive, then $T$ is either completely hyperexpansive,or, there exists no n-nilpotent operator, $n>1$, commuting with $T$. This is absurd.

(ii) Again, if $T$ is 2-expansive, then $T^{n}$ is 2-expansive for all positive integers $n$. Suppose that $T$ satisfies the commuting product property, i.e. assume $T^{2}$ is $(2+2-1=) 3$-expansive and $T^{n}$ is $n+1$-expansive. Then Theorem 2.2 implies that $T$ is $(n+1)$-hyperexpansive. This is false.

Additional hypotheses are required for results, similar to those for $(m, X)$-isometries, on products and perturbation by commuting nilpotents of $m$-expansive operators .

Given operators $A_{i}, B_{i}, X \in B(X)$ such that $\left(A_{i}, B_{i}\right) \in\left(m_{i}, X\right)$-expansive, $1 \leq i \leq 2$, we say in the following that:

a sequence $\left\{X_{j}\right\}_{j=r}^{r+k}=\left\{X_{j}\left(A_{1}, A_{2}, B_{1}, B_{2}, X\right)\right\}_{j=r}^{k+r}$ is a $(k+1)$-partial expansive sequence for $\left(A_{i}, B_{i}\right), i=1$ or 2 (exclusive or), if $\triangle_{A_{i}, B_{i}}\left(X_{j}\right) \leq 0$ for all $r \leq j \leq r+k$.

Partial hyperexpansive sequences occur naturally. Thus, if $(A, B) \in(m, X)$-hyperexpansive, then, with $\left(A_{1}, B_{1}\right)=(A, B),\left(A_{2}, B_{2}\right)=(I, I)$,

$$
\left\{X_{j}\right\}_{j=0}^{m-1}=\left\{\triangle_{A, B}^{j}(X)\right\}_{j=0}^{m-1}
$$

is an $m$-partial hyperexpansive sequence for $(A, B)$. Again, if $(A, B) \in$ completely- $(m, X)$-hyperexpansive, then $\left(\triangle_{A, B}^{j}(X) \leq 0\right.$ for all $j \geq 1$, hence $)$

$$
\left\{X_{j}\right\}_{j=m_{0}}^{k}=\left\{\triangle_{A, B}^{j-m_{0}}(X)\right\}_{j=m_{0}}^{k}
$$

is a $\left(k-m_{0}+1\right)$-partial hyperexpansive sequence for $(A, B)$ for all integers $m_{0} \geq 0$ and $k \geq m_{0}$. For $(m, X)$-left invertible pairs $(A, B)$,

$$
\left\{X_{j}\right\}_{j=0}^{k}=\left\{\triangle_{A, B}^{m-1+j}(X)\right\}_{j=0}^{k}
$$

is a $(k-m+1)$-partial hyperexpansive sequence for $(A, B)$ for all non-negative integers $k$. Finally, if $A_{i}, B_{i}$ and $X$ are the operators of equation (2), then the sequence

$$
\left\{\triangle_{A_{1}, B_{1}}^{m_{1}+j}(X)\right\}_{j=0}^{m_{2}-1}
$$

is an $m_{2}$-partial hyperexpansive sequence for $\left(A_{2}, B_{2}\right)$ and

$$
\left\{\triangle_{A_{2}, B_{2}}^{m_{2}+j}(X)\right\}_{j=0}^{m_{1}-1}
$$

is an $m_{1}$-partial hyperexpansive sequence for $\left(A_{1}, B_{1}\right)$.

The following theorem considers products of commuting expansive operators. 
Theorem 2.6. Given operators $A_{i}, B_{i}, P \in B(X), i=1,2$, such that $\left[A_{1}, A_{2}\right]=\left[B_{1}, B_{2}\right]=0$ and $\left(A_{i}, B_{i}\right) \in$ $\left(m_{i}, P\right)$-expansive, if

$$
\left\{X_{j}\right\}_{j=0}^{m_{1}-1}=\left\{\left(L_{A_{1}} R_{B_{1}}\right)^{m_{2}+j} \triangle_{A_{1}, B_{1}}^{m_{1}-1-j} \triangle_{A_{2}, B_{2}}^{m_{2}-1+j}(P)\right\}_{j=0}^{m_{1}-1}
$$

is an $m_{1}$-partial hyperexpansive sequence for $\left(A_{2}, B_{2}\right)$ and

$$
\left\{Y_{j}\right\}_{j=0}^{m_{2}-1}=\left\{\left(R_{A_{1}} R_{B_{1}}\right)^{m_{2}-1-j} \triangle_{A_{1}, B_{1}}^{m_{1}-1+j} \triangle_{A_{2}, B_{2}}^{m_{2}-1-j}(P)\right\}_{j=0}^{m_{2}-1}
$$

is an $m_{2}$-partial hyperexpansive sequence for $\left(A_{1}, B_{1}\right)$, then $\left(A_{1} A_{2}, B_{1} B_{2}\right) \in\left(m_{1}+m_{2}-1, P\right)$-expansive.

Proof. The commutativity hypotheses on $A_{i}$ and $B_{i}$ imply that $L_{A_{i}}$ and $R_{B_{i}}$ commute for all $i=1,2$, hence

$$
\begin{aligned}
& \triangle_{A_{1} A_{2}, B_{1} B_{2}}^{m_{1}+m_{2}-1}(P) \\
= & \left\{I-L_{A_{1} A_{2}} R_{B_{1} B_{2}}\right\}^{m_{1}+m_{2}-1}(P) \\
= & \left\{I-L_{A_{1}} L_{A_{2}} R_{B_{1}} R_{B_{2}}\right\}^{m_{1}+m_{2}-1}(P) \\
= & \left\{\triangle_{A_{1}, B_{1}}+L_{A_{1}} \triangle_{A_{2}, B_{2}} R_{B_{1}}\right\}^{m_{1}+m_{2}-1}(P) \\
= & \sum_{j=0}^{m_{1}+m_{2}-1}\left(\begin{array}{c}
m_{1}+m_{2}-1 \\
j
\end{array}\right)\left[\triangle_{A_{1}, B_{1}}^{j}\left(L_{A_{1}} \triangle_{A_{2}, B_{2}} R_{B_{1}}\right)^{m_{1}+m_{2}-1-j}\right](P) \\
= & \sum_{j=0}^{m_{1}-1}\left(\begin{array}{c}
m_{1}+m_{2}-1 \\
j
\end{array}\right)\left(L_{A_{1}} R_{B_{1}}\right)^{m_{1}+m_{2}-1-j} \triangle_{A_{2}, B_{2}}\left[\triangle_{A_{1}, B_{1}}^{j} \triangle_{A_{2}, B_{2}}^{m_{1}+m_{2}-2-j}\right](P)+ \\
+ & \sum_{j=m_{1}}^{m_{1}+m_{2}-1}\left(\begin{array}{c}
m_{1}+m_{2}-1 \\
j
\end{array}\right)\left(L_{A_{1}} R_{B_{1}}\right)^{m_{1}+m_{2}-1-j} \triangle_{A_{1}, B_{1}}\left[\triangle_{A_{1}, B_{1}}^{j-1} \triangle_{A_{2}, B_{2}}^{m_{1}+m_{2}-1-j}\right](P) .
\end{aligned}
$$

Defining the sequences $\left\{X_{j}\right\}_{j=0}^{m_{1}-1}$ and $\left\{Y_{j}\right\}_{j=0}^{m_{2}-1}$ as in the statement of the theorem, we have

$$
\begin{aligned}
& \triangle_{A_{2}, B_{2}}\left[X_{j}\right]_{j=0}^{m_{1}-1}=\left[\left(L_{A_{1}} R_{B_{1}}\right)^{m_{2}+j} \triangle_{A_{1}, B_{1}}^{m_{1}-1-j} \triangle_{A_{2}, B_{2}}^{m_{2}+j}(P)\right]_{j=0}^{m_{1}-1} \\
= & {\left[\left(L_{A_{1}} R_{B_{1}}\right)^{m_{1}+m_{2}-1-j} \triangle_{A_{1}, B_{1}}^{j} \triangle_{A_{2}, B_{2}}^{m_{1}+m_{2}-1-j}(P)\right]_{j=0}^{m_{1}-1} \text {, and } } \\
& \triangle_{A_{1}, B_{1}}\left[Y_{j}\right]_{j=0}^{m_{2}-1}=\left[\left(L_{A_{1}} R_{B_{1}}\right)^{m_{2}-1-j} \triangle_{A_{2}, B_{2}}^{m_{2}-1-j} \triangle_{A_{1}, B_{1}}^{m_{1}+j}(P)\right]_{j=0}^{m_{2}-1} \\
= & {\left[\left(L_{A_{1}} R_{B_{1}}\right)^{m_{1}+m_{2}-1-j} \triangle_{A_{1}, B_{1}}^{j} \triangle_{A_{2}, B_{2}}^{m_{1}+m_{2}-1-j}(P)\right]_{j=m_{1}}^{m_{1}+m_{2}-1} . }
\end{aligned}
$$

Since the sequences $\left\{X_{j}\right\}_{j=0}^{m_{1}-1}$ and $\left\{Y_{j}\right\}_{j=0}^{m_{2}-1}$ are hyperexpansive for $\left(A_{2}, B_{2}\right)$ and $\left(A_{1}, B_{1}\right)$, respectively, all the entries in the final two sums above are less than or equal to zero. Hence the result.

Observe that if $A_{i}, B_{i}$ satisfy the commutativity hypotheses of the theorem and $\left(A_{i}, B_{i}\right) \in\left(m_{i}, P\right)$-left invertible, then $\left\{X_{j}\right\}_{j=0}^{m_{2}-1}$ and $\left\{Y_{j}\right\}_{j=0}^{m_{1}-1}$ are the $\{0\}$ sequence, hence $\left(A_{1} A_{2}, B_{1} B_{2}\right) \in\left(m_{1}+m_{2}-1\right)$-left invertible. In particular, if $A_{1}=B_{1}^{\star}, A_{2}=B_{2}^{\star},\left[B_{1}, B_{2}\right]=0,\left(B_{i}^{\star}, B_{i}\right) \in\left(m_{i}, P\right)$-isometic, then $\left(B_{1}^{\star} B_{2}^{\star}, B_{1} B_{2}\right) \in\left(m_{1}+m_{2}-1, P\right)$ isometric $[8,16,27]$.

If $N \in B(X)$ is an $n$-nilpotent which commutes with $B \in B(X)$, then

$$
\begin{aligned}
& \triangle_{A, B+N}^{m+n-1}(P)=\left[\left(I-L_{A} R_{B}\right)+L_{A} R_{N}\right]^{m+n-1}(P) \\
& =\left[\sum_{j=0}^{m+n-1}\left(\begin{array}{c}
m+n-1 \\
j
\end{array}\right) \triangle_{A, B}^{j}\left(L_{A} R_{N}\right)^{m+n-1-j}\right](P) \\
& =\left[\sum_{j=m}^{m+n-1}\left(\begin{array}{c}
m+n-1 \\
j
\end{array}\right) \triangle_{A, B}^{j}\left(L_{A} R_{N}\right)^{m+n-1-j}\right](P)
\end{aligned}
$$


for all $A \in B(X)$ (since $N^{t}=0$ for all $t \geq n$ and $j \leq m-1$ implies $m+n-1-j \geq n$ ). Define the sequence $\left\{X_{j}\right\}_{j=m}^{m+n-1}$ by setting

$$
\triangle_{A, B}^{m-1-j}\left(L_{A} R_{N}\right)^{n-1-j}(P)=X_{j},
$$

and assume that $(A, B) \in(m, P)$-expansive with $\left\{X_{j}\right\}_{j=0}^{n-1}$ an $n$-partial hyperexpansive sequence for $(A, B)$. Then $(A, B+N) \in(m+n-1, P)$-expansive. For operators $T, N \in B(\mathcal{H}),[N, T]=0$ and $N^{n}=0$, such that $\left(T^{\star}, T\right) \in(m, P)$-expansive, this takes the following form.

Theorem 2.7. Given operators $T, N, P \in B(\mathcal{H})$ such that $[N, T]=0, N^{n}=0$ and $\left(T^{\star}, T\right) \in(m, P)$-expansive, $a$ sufficient condition for $\left(T^{\star}+N^{\star}, T+N\right) \in(m+2 n-2, P)$-expansive is that

$$
\left\{X_{j}\right\}_{j=0}^{2 n-2}=\left\{\triangle_{T^{*}, T}^{m-1+j} S^{2 n-2-j}(P)\right\}_{j=0}^{2 n-2}, S=L_{N^{*}} R_{T}+\left(L_{T^{*}}+R_{N^{*}}\right) R_{N},
$$

is an $(2 n-1)$-partial hyperexpansive sequence for $\left(T^{\star}, T\right)$.

Proof. Defining the operator $S$ as in the statement of the theorem, we have

$$
\triangle_{T^{*}+N^{*}, T+N}(P)=\left(\triangle_{T^{*}, T}+S\right)(P),
$$

and hence

$$
\triangle_{T^{*}+N^{*}, T+N}^{m+2 n-2}(P)=\left[\sum_{j=0}^{m+2 n-2}\left(\begin{array}{c}
m+2 n-2 \\
j
\end{array}\right) \triangle_{T^{\star}, T}^{j} S^{m+2 n-2-j}\right](P) .
$$

Since

$$
S^{m+2 n-2-j}=\sum_{k=0}^{m+2 n-2-j}\left(\begin{array}{c}
m+2 n-2-j \\
k
\end{array}\right)\left(L_{N^{*}} R_{T}\right)^{m+2 n-2-j-k}\left(L_{T^{*}}+R_{N^{*}}\right)^{k} R_{N}^{k},
$$

and $N^{t}=0$ for all $t \geq n$,

$$
S^{m+2 n-2-j}=0 \text { for all } k \geq n .
$$

Assume now that $k \leq n-1$, and consider $L_{N^{*}}^{m+2 n-2-j-k}$. Since $m+2 n-2-j-k \geq m+n-1-j$ and $n \leq m+n-1-j$ if and only if $j \leq m-1$,

$$
\begin{aligned}
\triangle_{T^{*}+N^{*}, T+N}^{m+2 n-2}(P) & =\sum_{j=0}^{m+2 n-2}\left(\begin{array}{c}
m+2 n-2 \\
j
\end{array}\right) \triangle_{T^{*}, T}^{j} S^{m+2 n-2-j}(P) \\
& =\sum_{j=m}^{m+2 n-2}\left(\begin{array}{c}
m+2 n-2 \\
j
\end{array}\right)\left[\triangle_{T^{*}, T}^{j} S^{m+2 n-2-j}\right](P) .
\end{aligned}
$$

Since

$$
\left\{\triangle_{T^{*}, T}^{m-1+j} S^{2 n-2-j}\right\}_{j=0}^{2 n-2}=\left\{\triangle_{T^{*}, T^{j-1}} S^{m+2 n-2-j}\right\}_{j=m}^{m+2 n-2},
$$

and

$$
\triangle_{T^{*}, T}\left[\triangle_{T^{*}, T}^{j-1} S^{m+2 n-2-j}(P)\right] \leq 0
$$

for all $m \leq j \leq m+2 n-2$, all the entries in the sum are less than or equal to zero. This completes the proof.

It is clear that

$$
\triangle_{T^{*}, T}^{j} S^{m+2 n-2-j}(P)=S^{m+2 n-2-j} \triangle_{T^{*}, T}^{j}(P),
$$

hence if $T$ is $(m, P)$-isometric, then $T+N$ is $(m+2 n-2, P)$-isometric. 


\section{Drazin invertible $(\boldsymbol{m}, \boldsymbol{P})$-expansive operators}

A straightforward induction argument proves that

$$
(a-1)^{m}=a^{m}-\sum_{j=0}^{m-1}\left(\begin{array}{c}
m \\
j
\end{array}\right)(a-1)^{j} .
$$

If we let $a=L_{A} R_{B}$ (and think of 1 as the identity operator), then

$$
\begin{aligned}
\tilde{\triangle}_{A, B}^{m}(P) & =\left(L_{A} R_{B}-I\right)^{m}(P) \\
& =\left(L_{A} R_{B}\right)^{m}-\sum_{j=0}^{m-1}\left(\begin{array}{c}
m \\
j
\end{array}\right) \tilde{\triangle}_{A, B}^{j}(P) \\
& =(-1)^{m} \triangle_{A, B}^{m}(P)
\end{aligned}
$$

for all $P \in B(X)$. Evidently,

$$
\tilde{\triangle}_{A, B}^{j}=L_{A} \tilde{\triangle}_{A, B}^{j-1} R_{B}-\tilde{\triangle}_{A, B}^{j-1} .
$$

Hence, for all integers $n \geq m$,

$$
\begin{aligned}
& \sum_{j=0}^{m-1}\left(\begin{array}{c}
n \\
j
\end{array}\right) L_{A} \tilde{\triangle}_{A, B}^{j} R_{B} \\
= & \sum_{j=0}^{m-1}\left(\begin{array}{c}
n \\
j
\end{array}\right) \tilde{\triangle}_{A, B}^{j+1}+\sum_{j=0}^{m-1}\left(\begin{array}{c}
n \\
j
\end{array}\right) \tilde{\triangle}_{A, B}^{j} \\
= & \left(\begin{array}{c}
n \\
m-1
\end{array}\right) \tilde{\triangle}_{A, B}^{m}+\sum_{j=0}^{m-1}\left(\begin{array}{c}
n+1 \\
j
\end{array}\right) \tilde{\triangle}_{A, B}^{j} .
\end{aligned}
$$

Consider now $\left(L_{A} R_{B}\right)^{t} \tilde{\triangle}_{A, B}^{m}(P)$, where $A, B, P \in B(\mathcal{H})$. If $t=1$, then equation (5) implies

$$
\begin{aligned}
& L_{A} R_{B} \widetilde{\triangle}_{A, B}^{m}(P)=\left(L_{A} R_{B}\right)^{m+1}(P)-\sum_{j=0}^{m-1}\left(\begin{array}{c}
m \\
j
\end{array}\right) L_{A} R_{B} \tilde{\triangle}_{A, B}^{j}(P) \\
& =\left(L_{A} R_{B}\right)^{m+1}(P)-\left[\sum_{j=0}^{m-1}\left(\begin{array}{c}
m \\
j
\end{array}\right) \tilde{\triangle}_{A, B}^{j+1}(P)+\sum_{j=0}^{m-1}\left(\begin{array}{c}
m \\
j
\end{array}\right) \tilde{\triangle}_{A, B}^{j}(P)\right] \\
& =\left(L_{A} R_{B}\right)^{m+1}(P)-\left[\left(\begin{array}{c}
m \\
m-1
\end{array}\right) \tilde{\triangle}_{A, B}^{m}(P)+\sum_{j=0}^{m-1}\left(\begin{array}{c}
m+1 \\
j
\end{array}\right) \tilde{\triangle}_{A, B}^{j}(P)\right] \text {, } \\
& \left(L_{A} R_{B}\right)^{2} \tilde{\triangle}_{A, B}^{m}(P)=\left(L_{A} R_{B}\right)^{m+2}(P)-\left(\begin{array}{c}
m \\
m-1
\end{array}\right) L_{A} R_{B} \tilde{\triangle}_{A, B}^{m}(P) \\
& -\sum_{j=0}^{m-1}\left(\begin{array}{c}
m+1 \\
j
\end{array}\right) \tilde{\triangle}_{A, B}^{j+1}(P)-\sum_{j=0}^{m-1}\left(\begin{array}{c}
m+1 \\
j
\end{array}\right) \tilde{\triangle}_{A, B}^{j}(P) \\
& =\left(L_{A} R_{B}\right)^{m+2}(P)-\left[\sum_{k=0}^{1}\left(\begin{array}{c}
m+1-k \\
m-1
\end{array}\right)\left(L_{A} R_{B}\right)^{k}\right] \tilde{\triangle}_{A, B}^{m}(P)- \\
& -\sum_{j=0}^{m-1}\left(\begin{array}{c}
m+2 \\
j
\end{array}\right) \tilde{\triangle}_{A, B}^{j}(P) \text {, }
\end{aligned}
$$




$$
\begin{aligned}
\left(L_{A} R_{B}\right)^{3} \tilde{\triangle}_{A, B}^{m}(P)= & \left(L_{A} R_{B}\right)^{m+3}(P)-\left[\sum_{k=0}^{1}\left(\begin{array}{c}
m+1-k \\
m-1
\end{array}\right)\left(L_{A} R_{B}\right)^{k+1}\right] \tilde{\triangle}_{A, B}^{m}(P)- \\
& -\sum_{j=0}^{m-1}\left(\begin{array}{c}
m+2 \\
j
\end{array}\right) L_{A} R_{B} \tilde{\triangle}_{A, B}^{j}(P) \\
= & \left(L_{A} R_{B}\right)^{m+3}(P)-\left[\sum_{k=0}^{2}\left(\begin{array}{c}
m+1-k \\
m-1
\end{array}\right)\left(L_{A} R_{B}\right)^{k}\right] \tilde{\triangle}_{A, B}^{m}(P)- \\
& -\sum_{j=0}^{m-1}\left(\begin{array}{c}
m+3 \\
j
\end{array}\right) \tilde{\triangle}_{A, B}^{j}(P),
\end{aligned}
$$

and by a finite induction argument that

$$
\begin{aligned}
\left(L_{A} R_{B}\right)^{n-m} \tilde{\triangle}_{A, B}^{m}(P)= & \left(L_{A} R_{B}\right)^{n}(P)-\left[\sum_{k=0}^{n-m-1}\left(\begin{array}{c}
n+1-k \\
m-1
\end{array}\right)\left(L_{A} R_{B}\right)^{k}\right] \tilde{\triangle}_{A, B}^{m}(P)- \\
& -\sum_{j=0}^{m-1}\left(\begin{array}{c}
n \\
j
\end{array}\right) \tilde{\triangle}_{A, B}^{j}(P)
\end{aligned}
$$

for all integers $n \geq m$.

Proposition 3.1. Given operators $A, B, P \in B(\mathcal{H})$, if the triplet $\left(A, B, \tilde{\triangle}_{A, B}^{m}(P)\right)$ preserves order and $\tilde{\triangle}_{A, B}^{m}(P) \leq$ 0 , then

$$
\left(L_{A} R_{B}\right)^{n}(P) \leq \sum_{j=0}^{m-1}\left(\begin{array}{c}
n \\
j
\end{array}\right) \tilde{\triangle}_{A, B}^{j}(P)
$$

for all integers $n \geq m$.

Proof. If the triplet $\left(A, B, \tilde{\triangle}_{A, B}^{m}(P)\right)$ preserves order and $\tilde{\triangle}_{A, B}^{m}(P) \leq 0$, then inequality (6) follows from

$$
\begin{aligned}
& \left(L_{A} R_{B}\right)^{n}(P)-\sum_{j=0}^{m-1}\left(\begin{array}{c}
n \\
j
\end{array}\right) \tilde{\triangle}_{A, B}^{j}(P) \\
= & \left(L_{A} R_{B}\right)^{n-m} \tilde{\triangle}_{A, B}^{m}(P)+\sum_{k=0}^{n-m-1}\left(\begin{array}{c}
n+1-k \\
m-1
\end{array}\right) \tilde{\triangle}_{A, B}^{m}(P) \\
\leq & 0 .
\end{aligned}
$$

If we now further assume in Proposition 3.1 that the triplet $(A, B, P), P \geq 0$, preserves order, then inequality (6) implies

$$
\begin{aligned}
(0 \leq)\left(L_{A} R_{B}\right)^{n}(P) & \leq \sum_{j=0}^{m-1}\left(\begin{array}{c}
n \\
j
\end{array}\right) \tilde{\triangle}_{A, B}^{j}(P) \\
& =\left(\begin{array}{c}
n \\
m-1
\end{array}\right) \tilde{\triangle}_{A, B}^{m-1}(P)+\sum_{j=0}^{m-2}\left(\begin{array}{c}
n \\
j
\end{array}\right) \tilde{\triangle}_{A, B}^{j}(P) .
\end{aligned}
$$

Hence

$$
(0 \leq) \frac{1}{n^{m-1}}\left(L_{A} R_{B}\right)^{n}(P) \leq \frac{1}{n^{m-1}}\left[\left(\begin{array}{c}
n \\
m-1
\end{array}\right) \tilde{\triangle}_{A, B}^{m-1}(P)+\sum_{j=0}^{m-2}\left(\begin{array}{c}
n \\
j
\end{array}\right) \tilde{\triangle}_{A, B}^{j}(P)\right] .
$$


Since $\left(\begin{array}{c}n \\ m-1\end{array}\right)$ is of the order $n^{m-1}$ and $\left(\begin{array}{c}n \\ m-2\end{array}\right)$ is of the order $n^{m-2}$ for large $n$, letting $n \longrightarrow \infty$ we have

$$
\tilde{\triangle}_{A, B}^{m-1}(P) \geq 0\left(\Longleftrightarrow(-1)^{m} \triangle_{A, B}^{m-1}(P) \leq 0\right) .
$$

Given invertible operators $A, B \in B(\mathcal{H})$, we say that the triplet $\left(A, B, X_{S}\right)$ is doubly order preserving if the triplets $\left(A, B, X_{S}\right)$ and $\left(A^{-1}, B^{-1}, X_{S}\right)$ are order preserving.

It is clear from inequality (7) that if $m$ is an odd integer and $\triangle_{A, B}^{m}(P) \geq 0$, i.e. $(A, B) \in(m, P)$-contractive, then $\widetilde{\triangle}_{A, B}^{m}(P) \leq 0$ and $\tilde{\triangle}_{A, B}^{m-1}(P)=\triangle_{A, B}^{m-1}(P) \geq 0$. The following theorem says that for invertible $(m, P)$-expansive pairs $(A, B)$ satisfying a suitable order preserving property, either $(A, B) \in(m-1, P)$-isometric or $(A, B) \in$ $(m-1, P)$-contractive.

Theorem 3.2. Given operators $A, B, P \in B(\mathcal{H})$ such that $A$ and $B$ are invertible, $P \geq 0$ and $(A, B) \in(m, P)$ expansive, if the triplet $(A, B, X)$ doubly preserves order for $X=\triangle_{A, B}^{t}(P), t=0, m-1$ and $m$, then

$$
\begin{aligned}
& (A, B) \text { and }\left(A^{-1}, B^{-1}\right) \in(m-1, P)-\text { isometric if } m \text { is even, } \\
& (A, B) \text { and }\left(A^{-1}, B^{-1}\right) \in(m-1, P)-\text { contractive if } m \text { is odd. }
\end{aligned}
$$

Proof. We consider $m$ even and $m$ odd cases separately.

$m$ even. If $m$ is even, then

$$
\triangle_{A, B}^{m}(P) \leq 0 \Longleftrightarrow \widetilde{\triangle}_{A, B}^{m}(P) \leq 0,
$$

and this, since $\left(A, B, \triangle_{A, B}^{m}(P)\right)$ and $(A, B, P)$ preserve order, implies

$$
\tilde{\triangle}_{A, B}^{m-1}(P) \geq 0 \Longleftrightarrow \triangle_{A, B}^{m-1}(P) \leq 0
$$

(see above). Since $A, B$ are invertible and $\left(A^{-1}, B^{-1}, \triangle_{A, B}^{m}(P)\right)$ also preserves order,

$$
\triangle_{A, B}^{m}(P) \leq 0 \Longleftrightarrow\left[\left(L_{A} R_{B}\right)^{-m} \triangle_{A, B}^{m}\right](P) \leq 0 \Longleftrightarrow \tilde{\triangle}_{A^{-1}, B^{-1}}^{m}(P) \leq 0 .
$$

Consequently, since $\left(A^{-1}, B^{-1}, \triangle_{A, B}^{m-1}(P)\right)$ preserves order,

$$
\tilde{\triangle}_{A^{-1}, B^{-1}}^{m-1}(P) \geq 0 \Longleftrightarrow\left[\left(L_{A^{-1}} R_{B^{-1}}\right)^{m-1} \tilde{\triangle}_{A, B}^{m-1}\right](P) \geq 0 \Longleftrightarrow \triangle_{A, B}^{m-1}(P) \geq 0 .
$$

Combining, $\triangle_{A, B}^{m-1}(P)=0$ (which then implies $(A, B) \in(n, P)$-isometric for all integers $n \geq m-1$ ).

$m$ odd. If $m$ is odd, and both $\left(A, B, \triangle_{A, B}^{m}(P)\right)$ and $(A, B, P)$ doubly preserves order, then

$$
\triangle_{A, B}^{m}(P) \leq 0 \Longleftrightarrow \tilde{\triangle}_{A^{-1}, B^{-1}}^{m}(P) \leq 0,
$$

and this, since $m-1$ is even and $\left(A, B, \triangle_{A, B}^{m-1}(P)\right)$ doubly preserves order, in turn implies

$$
\begin{aligned}
& \tilde{\triangle}_{A^{-1}, B^{-1}}^{m-1}(P) \geq 0 \Longleftrightarrow \triangle_{A^{-1}, B^{-1}}^{m-1}(P) \geq 0 \\
\Longleftrightarrow & \widetilde{\triangle}_{A, B}^{m-1}(P) \geq 0 \Longleftrightarrow \triangle_{A, B}^{m-1}(P) \geq 0 .
\end{aligned}
$$

This completes the proof.

Drazin Invertible Pairs. Recall [29] that $A \in B(X)$ is Drazin invertible, with Drazin inverse $A_{d}$ (of order $p$ for some positive integer $p$ ) if

$$
\left[A_{d}, A\right]=0, A_{d}^{2} A=A_{d}, A^{p+1} A_{d}=A^{p} .
$$


he Drazin inverse $A_{d}$ of $A$, whenever it exists, is unique. The Drazin invertibility of $A$ implies that 0 is an isolated point of the spectrum of $A$ and there exists a direct sum decomposition

$$
X=A^{p}(X) \oplus A^{-p}(X)=X_{1} \oplus X_{2}
$$

of $X$ and a direct sum decomposition

$$
A=\left.\left.A\right|_{x_{1}} \oplus A\right|_{X_{2}}=A_{1} \oplus A_{2}
$$

of $A$, where $A_{1}$ is invertible and $A_{2}$ is $p$-nilpotent. It is easily seen that

$$
A_{d}=A_{1}^{-1} \oplus 0 \in B\left(X_{1} \oplus X_{2}\right) .
$$

The following theorem extends Theorem 3.2 to Drazin invertible pairs $(A, B)$ to prove that if $A=A_{1} \oplus A_{2} \in$ $B\left(\mathcal{H}_{1} \oplus \mathcal{H}_{2}\right), B=B_{1} \oplus B_{2} \in B\left(\mathcal{H}_{1}^{\prime} \oplus \mathcal{H}_{2}^{\prime}\right),(A, B) \in(m, P)$-expansive and the triplet $\left(A_{1}, B_{1}, X\right), X=\tilde{\triangle}_{A_{1}, B_{1}}^{t}\left(P_{11}\right)$ for $t=m, m-1,0$ and $P_{11}=\left.P\right|_{\left(\mathcal{H}_{1}^{\prime} \rightarrow \mathcal{H}_{1}\right)}$, is doubly oredr preserving, then either $(A, B) \in(m-1, P)$-isometric or $(A, B) \in(m-1, P)$-contractive.

Theorem 3.3. Given operators $A, B, P \in B(\mathcal{H})$ such that $P \geq 0, A$ and $B$ are Drazin invertible with Drazin inverses $A_{d}$ and $B_{d}$, if $(A, B) \in(m, P)$-expansive and the triplets $(A, B, X)$ and $\left(A_{d}, B_{d}, X\right), X=\tilde{\triangle}_{A, B}^{t}(P)$ for $t=m, m-1$ and 0 , are order preserving, then $(A, B) \wedge\left(A_{d}, B_{d}\right) \in(m-1, P)$-isometric if $m$ is even and $(A, B) \wedge$ $\left(A_{d}, B_{d}\right) \in(m-1, P)$-contractive if $m$ is odd.

Proof. Letting $A, B, A_{d}, B_{d}$ have the (canonical) representations

$$
\begin{aligned}
& A=A_{1} \oplus A_{2} \in B\left(\mathcal{H}_{1} \oplus \mathcal{H}_{2}\right), A_{1} \text { invertible and } A_{2}^{p}=0, \\
& B=B_{1} \oplus B_{2} \in B\left(\mathcal{H}^{\prime}{ }_{1} \oplus \mathcal{H}^{\prime}{ }_{2}\right), B_{1} \text { invertible and } B_{2}^{q}=0, \\
& A_{d}=A_{1}^{-1} \oplus 0 \in B\left(\mathcal{H}_{1} \oplus \mathcal{H}_{2}\right), B_{d}=B_{1}^{-1} \oplus 0 \in B\left(\mathcal{H}^{\prime}{ }_{1} \oplus \mathcal{H}^{\prime}{ }_{2}\right)
\end{aligned}
$$

for some positive integers $p$ and $q$, let $P \in B\left(\mathcal{H}^{\prime}{ }_{1} \oplus \mathcal{H}^{\prime}{ }_{2}, \mathcal{H}_{1} \oplus \mathcal{H}_{2}\right)$ have the matrix representation $P=\left[P_{i j}\right]_{i, j=1}^{2}$. Then

$$
\triangle_{A, B}^{m}(P)=\left[\sum_{k=0}^{m}(-1)^{k}\left(\begin{array}{c}
m \\
k
\end{array}\right) A_{i}^{k} P_{i j} B_{j}^{k}\right]_{i, j=1}^{2} \leq 0 .
$$

We prove that $P_{i j}=0$ for all $i=j \neq 1$, and to this end we start by considering

$$
\sum_{k=0}^{m}(-1)^{k}\left(\begin{array}{c}
m \\
k
\end{array}\right) A_{2}^{k} P_{22} B_{2}^{k} \leq 0
$$

Let $r=\max (p, q)$; then $A_{2}^{r}=B_{2}^{r}=0$. Since $\left(A, B, \tilde{\triangle}_{A, B}^{m}(P)\right)$ is order preserving, multiplying $\triangle_{A, B}^{m}(P)$ by $A^{r-t}$ on the left and by $B^{r-t}$ on the right, we have:

if $t=1$, then

$$
A_{2}^{r-1}\left(\sum_{k=0}^{m}(-1)^{k}\left(\begin{array}{c}
m \\
k
\end{array}\right) A_{2}^{k} P_{22} B_{2}^{k}\right) B_{2}^{r-1} \leq 0 \Longrightarrow A_{2}^{r-1} P_{22} B_{2}^{r-1} \leq 0
$$

if $t=2$, then

$$
\begin{aligned}
& A_{2}^{r-2} P_{22} B_{2}^{r-2}-\left(\begin{array}{c}
m \\
1
\end{array}\right) A_{2}^{r-1} P_{22} B_{2}^{r-1} \leq 0 \\
\Rightarrow & A_{2}^{r-2} P_{22} B_{2}^{r-2} \leq m A_{2}^{r-1} P_{22} B_{2}^{r-1} \leq 0 ;
\end{aligned}
$$

if $t=3$, then

$$
A_{2}^{r-3} P_{22} B_{2}^{r-3}-\left(\begin{array}{c}
m \\
1
\end{array}\right) A_{2}^{r-2} P_{22} B_{2}^{r-2}+\left(\begin{array}{c}
m \\
2
\end{array}\right) A_{2}^{r-1} P_{22} B_{2}^{r-1} \leq 0
$$




$$
\begin{aligned}
& \Rightarrow A_{2}^{r-3} P_{22} B_{2}^{r-3} \leq\left(\begin{array}{c}
m \\
1
\end{array}\right) A_{2}^{r-2} P_{22} B_{2}^{r-2}-\left(\begin{array}{c}
m \\
2
\end{array}\right) A_{2}^{r-1} P_{22} B_{2}^{r-1} \\
& \Rightarrow A_{2}^{r-3} P_{22} B_{2}^{r-3} \leq\left(m^{2}-\frac{m(m-1)}{2}\right) A_{2}^{r-1} P_{22} B_{2}^{r-1}= \\
& =\left(\begin{array}{c}
m+1 \\
2
\end{array}\right) A_{2}^{r-1} P_{22} B_{2}^{r-1} \leq 0
\end{aligned}
$$

and more generally, by an induction argument, that if $t=s$, then

$$
A_{2}^{r-s} P_{22} B_{2}^{r-s} \leq\left(\begin{array}{c}
m+s-2 \\
s-1
\end{array}\right) A_{2}^{r-1} P_{22} B_{2}^{r-1} \leq 0 .
$$

Conclusion:

$$
P_{22} \leq 0 \text {. }
$$

Since $P \geq 0$ implies $P_{22} \geq 0$,

$$
P_{22}=0 \text {. }
$$

Recall that a $B(\mathcal{H})$ operator $P=\left[P_{i j}\right]_{i, j=1}^{2} \geq 0$ if and only if $P_{11}, P_{22} \geq 0$ and $P_{12}^{\star}=P_{21}=P_{22}^{\frac{1}{2}} C P_{11}^{\frac{1}{2}}$ for some contraction $C$ [30]. This, since $P_{22}=0$, implies

$$
P_{12}=P_{21}=0 \Longrightarrow P=P_{11} \oplus 0
$$

and

$$
\triangle_{A, B}^{m}(P)=\triangle_{A_{1}, B_{1}}^{m}\left(P_{11}\right) \oplus 0 \leq 0 \Longrightarrow \triangle_{A_{1}, B_{1}}^{m}\left(P_{11}\right) \leq 0 .
$$

Evidently, $(A, B, X)$ and $\left(A_{d}, B_{d}, X\right)$ order preserving, $X$ as in the statement of the theorem, implies $\left(A_{1}, B_{1}, X_{1}\right)$ and $\left(A_{1}^{-1}, B_{1}^{-1}, X_{1}\right)$, where $X_{1}=\tilde{\triangle}_{A_{1}, B_{1}}^{t}\left(P_{11}\right)$ for $t=m, m-1,0$, are order preserving. Hence $\left(A_{1}, B_{1}, X_{1}\right)$ is doubly order preserving, Theorem 3.2 applies to the invertible pair $\left(A_{1}, B_{1}\right) \in\left(m, P_{11}\right)$ expansive to prove that

$$
\left(A_{1}^{-1}, B_{1}^{-1}\right) \wedge\left(A_{1}, B_{1}\right) \in\left\{\begin{array}{l}
\left(m-1, P_{11}\right)-\text { isometric if } m \text { is even } \\
\left(m-1, P_{11}\right)-\text { contractive if } m \text { is odd. }
\end{array}\right.
$$

Consequently, since $P=P_{11} \oplus 0$,

$$
\left(A_{d}, B_{d}\right) \wedge(A, B) \in\left\{\begin{array}{l}
(m-1, P)-\text { isometric if } m \text { is even } \\
(m-1, P)-\text { contractive if } m \text { is odd. }
\end{array}\right.
$$

This completes the proof.

A particularly important case of Theorem 3.2 and 3.3 is the one in which $A^{\star}=B=T:\left(T^{\star}, T, \tilde{\triangle}_{T^{*}, T}^{m}(P)\right)$ then preserves order (resp., doubly preserves order if $T$ is invertible) for all values of $m$. We have:

Corollary 3.4. Let $T, P \in B(\mathcal{H})$ be such that $P \geq 0$ and $\triangle_{T^{*}, T}^{m}(P) \leq 0$. If $T$ is invertible, respectively Drazin invertible, then

$$
\left(T^{\star^{-1}}, T^{-1}\right) \wedge\left(T^{\star}, T\right) \in\left\{\begin{array}{l}
(m-1, P)-\text { isometric if } m \text { is even } \\
(m-1, P)-\text { contractive if } m \text { is odd }
\end{array}\right.
$$

respectively

$$
\left(T_{d}^{\star}, T_{d}\right) \wedge\left(T^{\star}, T\right) \in\left\{\begin{array}{l}
(m-1, P)-\text { isometric if } m \text { is even } \\
(m-1, P)-\text { contractive if } m \text { is odd. }
\end{array}\right.
$$

In particular, if $m=2$ and $T$ is Drazin invertible, then $\left\|P^{\frac{1}{2}} T x\right\|=\left\|P^{\frac{1}{2}} x\right\|=\left\|P^{\frac{1}{2}} T_{d} x\right\|$. 
Here, the proof of the final assertion of the corollary is an immediate consequence of the fact that if $m=2$, then $T^{\star} P T=P=T_{d}^{\star} P T_{d}$.

Remark 3.5. (i). The argument of the proof of Theorem 3.3 leading to the conclusion $P_{12}=P_{21}=P_{22}=$ 0 for order preserving triplets $\left(A, B, \tilde{\triangle}_{A, B}^{m}(P)\right)$ implies that $(A, B) \notin(m, I)$-expansive for any pair $(A, B)$ of Drazin invertible operators (satisfying this order preserving property). In particular, there does not exist a Drazin invertible operator $T \in B(\mathcal{H})$ such that $\triangle_{T^{*}, T}^{m}(I) \leq 0$.

(ii) If a Banach space operator $T \in B(\mathcal{X})$ is expansive in the sense of (the definition in) equality (4), i.e., if $\triangle_{p}^{m}(P, T, x) \leq 0$ for all $x \in \mathcal{X}$, then

$$
\left\|P T^{n} x\right\|^{p} \leq \sum_{j=0}^{m-1}\left(\begin{array}{c}
n \\
j
\end{array}\right) \tilde{\triangle}_{p}^{j}(P, T, x)
$$

and if also $T$ is invertible, then

$$
\triangle_{p}^{m}(P, T, x) \leq 0 \Rightarrow\left\{\begin{array}{l}
\triangle_{p}^{m-1}(P, T, x)=\triangle_{p}^{m-1}\left(P, T^{-1}, x\right)=0 \text { if } m \text { is even } \\
\triangle_{p}^{m-1}(P, T, x) \wedge \triangle_{p}^{m-1}\left(P, T^{-1}, x\right) \geq 0 \text { if } m \text { is odd. }
\end{array}\right.
$$

Here, for the proof of (8) one argues as in the proof of Proposition 2.1 of Bayart [7] - simply replace $\left\|T^{k} x\right\|^{p}$ by $\left\|P T^{k} x\right\|^{p}$ to prove

$$
\tilde{\triangle}_{p}^{m}(P, T, x)=\left\|P T^{n} x\right\|^{p}-\sum_{j=0}^{m-1}\left(\begin{array}{c}
n \\
j
\end{array}\right) \tilde{\triangle}_{p}^{j}(P, T, x)
$$

for all integers $n \geq 0$. Evidently

$$
\begin{aligned}
& \sum_{j=0}^{m-1}\left(\begin{array}{c}
n \\
j
\end{array}\right) \tilde{\triangle}_{p}^{j}(P, T, T x)= \\
= & \left(\begin{array}{c}
n \\
m-1
\end{array}\right) \tilde{\triangle}_{p}^{m}(P, T, x)+\sum_{j=0}^{m-1}\left(\begin{array}{c}
n+1 \\
j
\end{array}\right) \tilde{\triangle}_{p}^{j}(P, T, x)
\end{aligned}
$$

Combine (10) with (11), and use a limit argument similar to that used to reach inequality (7), to obtain $\tilde{\triangle}_{p}^{m-1}(P, T, x) \geq 0$. Since $\tilde{\triangle}_{p}^{m-1}(P, T, x)=(-1)^{m-1} \triangle_{p}^{m-1}(P, T, x)$ and $\tilde{\triangle}_{p}^{m-1}\left(P, T^{-1}, x\right)=$ $(-1)^{m-1} \tilde{\triangle}_{p}^{m-1}\left(P, T, T^{-m+1} x\right)$, we also have that

$$
(-1)^{m-1} \tilde{\triangle}_{p}^{m-1}\left(P, T^{-1}, x\right)=\triangle_{p}^{m-1}\left(P, T^{-1}, x\right) \geq 0 .
$$

This proves (9). The case $P=I$ of (8) and (9) was first proved by Gu [21].

The following theorem proves that Theorem 3.3 extends to Drazin invertible Banach space operators $T$ satisfying $\triangle_{p}^{m}(P, T, x) \leq 0$.

Theorem 3.6. Given operators $P, T \in B(X)$ such that $T$ Drazin invertible with Drazin inverse $T_{d}$,

$$
\triangle_{p}^{m}(P, T, x) \leq 0 \Rightarrow\left\{\begin{array}{l}
\triangle_{p}^{m-1}(P, T, x) \wedge \triangle_{p}^{m-1}\left(P, T_{d}, x\right)=0 \text { if } m \text { is even } \\
\triangle_{p}^{m-1}(P, T, x) \wedge \triangle_{p}^{m-1}\left(P, T_{d}, x\right) \geq 0 \text { if } m \text { is odd }
\end{array}\right.
$$

Proof. If $T$ is Drazin invertible with Drazin inverse $T_{d}$ of index $q$ for some integer $q \geq 1$, then there exists a decomposition $X=X_{1} \oplus X_{2}$ of $X$ such that

$$
X_{1}=T^{q}(X), X_{2}=T^{-q}(X), T=\left.\left.T\right|_{x_{1}} \oplus T\right|_{X_{2}}=T_{1} \oplus T_{2},
$$


where $T_{1}$ is invertible and $T_{2}^{q}=0$. Furthermore

$$
T_{d}=T_{1}^{-1} \oplus 0 \in B\left(X_{1} \oplus X_{2}\right) .
$$

Letting $P \in B\left(X_{1} \oplus X_{2}\right)$ have the matrix representation $P=\left[P_{i k}\right]_{i, k=1}^{2}$, and letting $x=0 \oplus x_{2} \in X_{1} \oplus X_{2}$, it then follows from $\left(\triangle_{p}^{m}(P, T, x) \leq 0\right.$ implies $) \triangle_{p}^{m}\left(P, T, T^{q-1} \chi\right) \leq 0$ that

$$
\begin{aligned}
& \sum_{j=0}^{m}(-1)^{j}\left(\begin{array}{c}
m \\
j
\end{array}\right)\left\|P T^{j+q-1} x\right\|^{p}=\left\|P T^{q-1} x\right\|^{p} \\
= & \left\|P_{12} T_{2}^{q-1} x_{2} \oplus P_{22} T_{2}^{q-1} x_{2}\right\|^{p} \leq 0 \\
\Longleftrightarrow & P_{12} T_{2}^{q-1} x_{2}=P_{22} T_{2}^{q-1} x_{2}=0 .
\end{aligned}
$$

Considering next $\triangle_{p}^{m}\left(P, T, T^{q-2} \chi\right)$, and then repeating the process, it is seen that

$$
\begin{aligned}
& \|P x\|^{p}=\left\|P_{12} x_{2} \oplus P_{22} x_{2}\right\|^{p}=0 \\
\Longleftrightarrow \quad P_{12} x_{2} & =P_{22} x_{2}=0 .
\end{aligned}
$$

Since this is true for all $x_{2} \in X_{2}$, we must have

$$
P_{12}=P_{22}=0 \Rightarrow P=\left(\begin{array}{ll}
P_{11} & 0 \\
P_{21} & 0
\end{array}\right) \in B\left(X_{1} \oplus X_{2}\right) .
$$

Consequently, for all $x \in X$,

$$
\begin{aligned}
\triangle_{p}^{m}(P, T, x) & =\sum_{j=0}^{m}(-1)^{j}\left(\begin{array}{c}
m \\
j
\end{array}\right)\left\|P T^{j} x\right\|^{p} \leq 0 \\
& \Longleftrightarrow \sum_{j=0}^{m}(-1)^{j}\left(\begin{array}{c}
m \\
j
\end{array}\right)\left\|P\left(T_{1} \oplus 0\right)^{j} x\right\|^{p} \leq 0 \\
& \sum_{j=0}^{m}(-1)^{j}\left(\begin{array}{c}
m \\
j
\end{array}\right)\left\|P\left(T_{1} \oplus I_{2}\right)^{j} x\right\|^{p} \leq 0 \\
& \left(I_{2} \text { the identity of } B\left(x_{2}\right)\right) \\
& \sum_{j=0}^{m}(-1)^{j}\left(\begin{array}{c}
m \\
j
\end{array}\right)\left\|P\left(T_{1}^{-1} \oplus I_{2}\right)^{m-j} x\right\|^{p} \leq 0 \\
& \left(\text { replace } x \text { by }\left(T_{1} \oplus I_{2}\right)^{-m} x\right) \\
\Longleftrightarrow & \widetilde{\triangle}_{p}^{m}\left(P, T_{d}, x\right)=\sum_{j=0}^{m}(-1)^{j}\left(\begin{array}{c}
m \\
j
\end{array}\right)\left\|P T_{d}^{m-j} x\right\|^{p} \leq 0 \\
\Longrightarrow & \tilde{\triangle}_{p}^{m-1}\left(P, T_{d}, x\right)=(-1)^{m-1} \triangle_{p}^{m-1}\left(P, T_{d}, x\right) \geq 0 \\
& (\text { see above }) \\
\Longleftrightarrow & \tilde{\triangle}_{p}^{m-1}\left(P, T_{1}^{-1} \oplus I_{2}, x\right) \geq 0 \\
\Longleftrightarrow & (-1)^{m-1} \tilde{\triangle}_{p}^{m-1}\left(P, T_{1} \oplus I_{2}, x\right) \geq 0 \\
& \left(\text { replace } x \text { by }\left(T_{1} \oplus I_{2}\right)^{m} x\right) \\
\Longleftrightarrow & \triangle_{p}^{m-1}(P, T, x) \geq 0 .
\end{aligned}
$$

Thus, if $m$ is even, then

$$
\triangle_{p}^{m-1}(P, T, x) \geq 0, \triangle_{p}^{m-1}\left(P, T_{d}, x\right) \leq 0,
$$

and if $m$ is odd, then

$$
\left\{\triangle_{p}^{m-1}(P, T, x)\right\} \wedge\left\{\triangle_{p}^{m-1}\left(P, T_{d}, x\right)\right\} \geq 0
$$


Already we have (from (7) and (8) that)

$$
\begin{aligned}
\tilde{\triangle}_{p}^{m-1}(P, T, x) \geq 0 & \Longleftrightarrow \tilde{\triangle}_{p}^{m-1}\left(P, T_{1}+I_{2}, x\right) \geq 0 \\
& \Longleftrightarrow \triangle_{p}^{m-1}\left(P, T_{1}^{-1}+I_{2}, x\right) \geq 0 \\
& \Longleftrightarrow \triangle_{p}^{m-1}\left(P, T_{d}, x\right) \geq 0,
\end{aligned}
$$

i.e., if $m$ is even, then

$$
\triangle_{p}^{m-1}(P, T, x) \leq 0, \triangle_{p}^{m-1}\left(P, T_{d}, x\right) \geq 0
$$

and if $m$ is odd, then

$$
\triangle_{p}^{m-1}(P, T, x) \wedge \triangle_{p}^{m-1}\left(P, T_{d}, x\right) \geq 0 .
$$

Putting together the even, and the odd, cases, the proof follows.

Acknowledgements: It is my pleasure to thank a referee for his notes on the original version of the manuscript. His remarks asking for clarity on the "preservation of order property" have led to the current formulation of "order preserving triplets".

Conflict of interest: No conflict of interested reported.

\section{References}

[1] J. Agler, Hypercontractions and subnormality, J. Operator Th. 13(1985), 203-217.

[2] J. Agler and M. Stankus, $m$-Isometric transformations of Hilbert space I, Integral Equat. Oper. Theory 21(1995), 383-420.

[3] J. Agler and M. Stankus, $m$-Isometric transformations of Hilbert space II, Integral Equat. Oper. Theory 23(1995), 1-48.

[4] J. Agler and M. Stankus, $m$-Isometric transformations of Hilbert space III, Integral Equat. Oper. Theory 24(1996), $379-421$.

[5] O.A.M. Sid Ahmed, Some properties of $m$-isometries and $m$-invertible operators in Banach spaces, Acta Math. Sci. Ser. B English Ed. 32(2012), 520-530.

[6] F. Bayart, $m$-isometries on Banach Spaces, Math. Nachr. 284(2011), 2141-2147.

[7] F. Botelho and J. Jamison, Isometric properties of elementary operators, Linear Alg. Appl. 432(2010), 357-365.

[8] T. Bermúdez, A. Martinón and J.N. Noda, Products of m-isometries, Linear Alg. Appl. 408(2013) 80-86.

[9] T. Bermúdez, A. Martinón and J.N. Noda, An isometry plus a nilpotent operator is an $m$-isometry, Applications, J. Math. Anal Appl. 407(2013) 505-512.

[10] T. Bermúdez, A. Martinón, V. Müller and J.N. Noda, Perturbation of $m$-isometries by nilpotent operators, Abstract and Applied Analysis, Volume 2014, Article ID 745479(6pages).

[11] B.P. Duggal and I.H. Kim, Structure of elementary operators defining $m$-left invertible, $m$-selfadjoint and related classes of operators, J. Math Anal Appl. 495(2021), 124718.

[12] B.P. Duggal and I.H. Kim, Structure of $n$-quasi left $m$-invertible and related classes of operators, Demonstratio Mathematica 53(2020), 249-268.

[13] B. P. Duggal, Tensor product of $n$-isometries, Linear Alg. Appl. 437(2012), 307-318.

[14] B.P. Duggal, Tensor product of $n$-isometries II, Functional Anal. Approx. and Computation 4:1(2012), 27-32.

[15] B.P. Duggal, Tensor product of $n$-isometries III, Functional Anal. Approx. and Computation 4:2(2012), 61-67.

[16] C. Gu, Structure of left $n$-invertible operators and their applications, Studia Math. 226(2015), 189-211.

[17] C. Gu, Elementary operators which are $m$-isometric, Linear Alg. Appl. 451(2014), 49-64.

[18] A. Athavale, On completely hyperexpansive operators, Proc. Amer. Math. Soc. 124(1996), 3745-3752.

[19] O.A.M. Sid Ahmed, On $A(m, p)$-expansive and $A(m, p)$-hyperexpansive operators on Banach spaces II, J. Math. Comput. Sci. 5(2015), 123-128.

[20] G. Exner, I. B. Jung and C. Li, On k-hyperexpansive operators, J. Math. Anal. Appl. 323(2006), 569-582.

[21] C. Gu, On $(m, p)$-expansive and $(m, p)$-contractive operators on Hilbert and Banach spaces, J. Math. Anal. Appl. 426(2015), 893-916.

[22] S. Jung, Y. Kim, E. Ko and J.E.Lee, m On ( $A, m)$-expansive operators, Studia Math. 213(2012), 3-23.

[23] V.M. Sholapurkar and A. Athavale, Completely and alternatively hyperexpansive operators, J. Operator Th. 43(2000), 43-68.

[24] B.P. Duggal and I.H. Kim, Left $m$-invertibility by the adjoint of Drazin inverse and $m$-selfadjointness of Hilbert space operators, Lin. Multilin. Algebra. (DOI: 10.1080/03081087.2020.1793880)

[25] S. Richter, Invariant subspaces of the Dirichlet shift, J. Reine Angew. Math. 81(1988), 265-220.

[26] C. Gu, Functional calculus for $m$-isometrise and related operators on Hilbert spaces and Banach spaces, Acta Sci. Math. (Szeged) 81(2015), 605-641. 
[27] B.P. Duggal and V. Müller, Tensor product of left $n$-invertible operators, Studia Math. 215(2)(2013), 113-125.

[28] Trieu Le, Algebraic properties of operator roots of polynomials, J. Math. Anal. Appl. 421(2015), 1238-1246.

[29] D.S. Djordjevic and V. Rakocevic, Lectures on Generalized Inverses, Faculty of Sciences and Mathematics, University of Nis, 2008.

[30] T. Ando, Topics on Operator Inequalities, Division of Applied Mathematics, Research Institute of Applied Electricity, Hokkaido University, Sapporo, Japan (1978). 